\title{
An optimization method for evaluating variations in paint coating thickness by infrared thermography
}

\author{
S. Mezghani ${ }^{\star}$, E. Perrin ${ }^{\star}$, V. Vrabie ${ }^{\star}$, J.L Bodnar ${ }^{\star \star}$, J. Marthe ${ }^{\star \star \star}$, B. Cauwe $e^{\star \star \star}$ \\ ${ }^{*}$ CReSTIC/Châlons, Université de Reims Champagne-Ardenne, Chaussée du port, 51000 Châlons-en- \\ Champagne, France \\ ** GRESPI/ECATHERM, Université de Reims Champagne-Ardenne, BP 1039, 51687 Reims, France \\ *** CRITT-MDTS, 3 Boulevard Jean Delautre - ZHT du Moulin Leblanc, 08000 Charleville-Mézières,
}

France

\begin{abstract}
This paper is focused on the optimization of the pulsed thermography inspection of coating thickness. To this aim, an analysis method was developed for evaluating variations of coating thickness using active infrared thermography. This method, based on a linear decomposition of thermographic surface response evolution, uses a Partial Least Squares Regression algorithm to identify different phenomena affecting the overall thermal responses. Results show that the proposed technique allows to overcome the experimental conditions (positions, intensity) of heating sources and to enhance the robustness of the pulsed thermography technique for coating thickness heterogeneity evaluation.
\end{abstract}

\section{Introduction}

Advances in IR camera and computer technology in the last decade have encouraged the use of Pulsed Thermography (PT) as an alternative nondestructive technique in several applications including industrial inspection [1] and evaluation of fatigue damages in materials [2], especially for coating thickness analysis [3]. Unfortunately, PT presents some limitations that hinder its application as its high sensitivity to external reflection and emissivity variations. However, the major drawback is the non-uniform heat excitation. This impacts directly the thermal signature of paint coating in both the heating and cooling stage. Furthermore, it can lead also to a local overheating on the sample. That's why the configuration of the irradiation sources is a prime factor in PT inspection. In order to resolve the above issues, several researchers have studied the temperature distribution during the thermography inspection and suggested some measures to reduce the non-uniform temperature distribution. Chatterjee and Tuli inhibited the influence of non-uniform heating using a Fourier transformation based image reconstruction algorithm [4]. Other methods are used to improve thermal contrast and to overcome to this external artifact like absolute thermal contrast [5], standard contrast [6], and modified differential absolute contrast [7]. In addition, some filtering techniques have been also used [8, 9]. Recently, an algorithm based on the Partial Least Square Regression (PLSR) has been used to automatically enhance the defect detection in single layered sample by partially eliminate the background noise [10]. The methods mentioned above cannot efficiently perform the thermal signature extraction for multilayered samples or the application of those techniques are restricted to specific cases such as estimate the defects.

In this paper, a new treatment approach is proposed to overcome the harmful effects of non-uniform heating in active infrared thermography test. It is based on a linear decomposition of the thermographic surface response evolution using a PLSR algorithm based on the SVD decomposition. The approach is then applied to coating layer thickness evaluation from thermographic response. Results show that the proposed technique allows to discard the influence of experimental conditions (positions, intensity) of heating sources and to enhance the robustness of the pulsed thermography technique for coating thickness heterogeneity evaluation.

\section{Materials and methods}

\subsection{Experimental set-up}

The experimental dispositive consists of a pulsed thermography bench [11] with a modified excitation consisting of four basic flash lamps with a power of 2000 Watt. One, two, three or all flashes can be used in the same time as excitation source (Fig. 1). The heating source and the IR camera are located on the same side of the sample to inspect. On the considered metal sample, black paint layers with different thickness were deposited with a commercially available spray. The heterogeneity of paint layer is represented by three steps which the thickness varies from 56 to $80 \mu \mathrm{m}$ thickness. Inspected surface was heated during a short duration $(\approx 5 \mathrm{~ms})$ which is enough to have a thermal change without causing 


\subsection{1/qirt.2016.088}

damage [12]. Once the pulse is delivered to the sample surface, the acquisition of thermal images is carried out straightaway using an FLIR® infrared camera (SC655, with a 280x120 pixel array, 200 frames per second).

Flashs

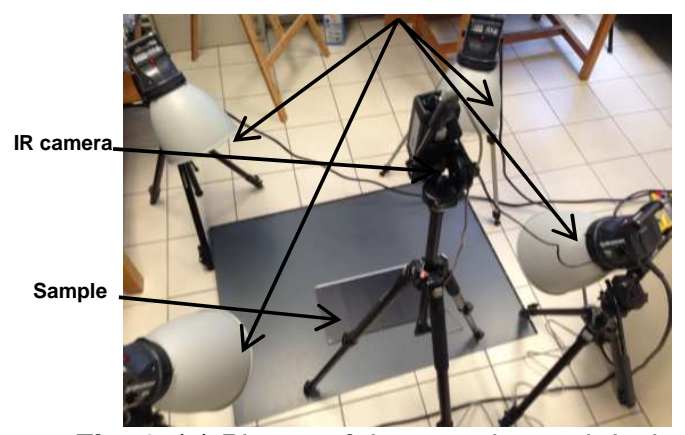

Fig. 1. (a) Picture of the experimental devices setup.

\subsection{Data acquisition and preprocessing}

At each acquisition time $t$, the thermal response on the sample is a thermal image, i.e. a two-dimensional data describing the spatial distribution of temperature. These temporal image sequences are stored into a cube structure of data, which is the stacking of the $N_{t}$ images of size $N_{x} \times N_{y}$. [13]. We can see in Fig. 2, the spatial heterogeneity of the energy deposited in the front of the surface. In fact, this non-uniformity is defined as a thermal pattern of a surface that varies from a uniform color tone when viewed with an infrared imaging system. This non-uniformity of the heating makes the analysis difficult, including the case where all four flashes are used since we cannot assure a perfect uniformity of the thermal excitation.

In the second step, the cube can be unfolded as a spatial-transient 2D vector $X$ with dimensions $N \times N_{t}$, where $N=N_{x} \times$ $N_{y}$. Then, a preprocessing operation is applied to this thermal response matrix to yields the thermal responses more sensitive to two principal parameters: deposited energy and the coating thickness. It is based on multiplying each row of the matrix with a time square root temporal function in accordance with the theoretical thermal response of bi-layered samples excited by a Dirac pulse excitation. Once this new 2D matrix is obtained, we apply the PLSR algorithm as detailed in the next section.
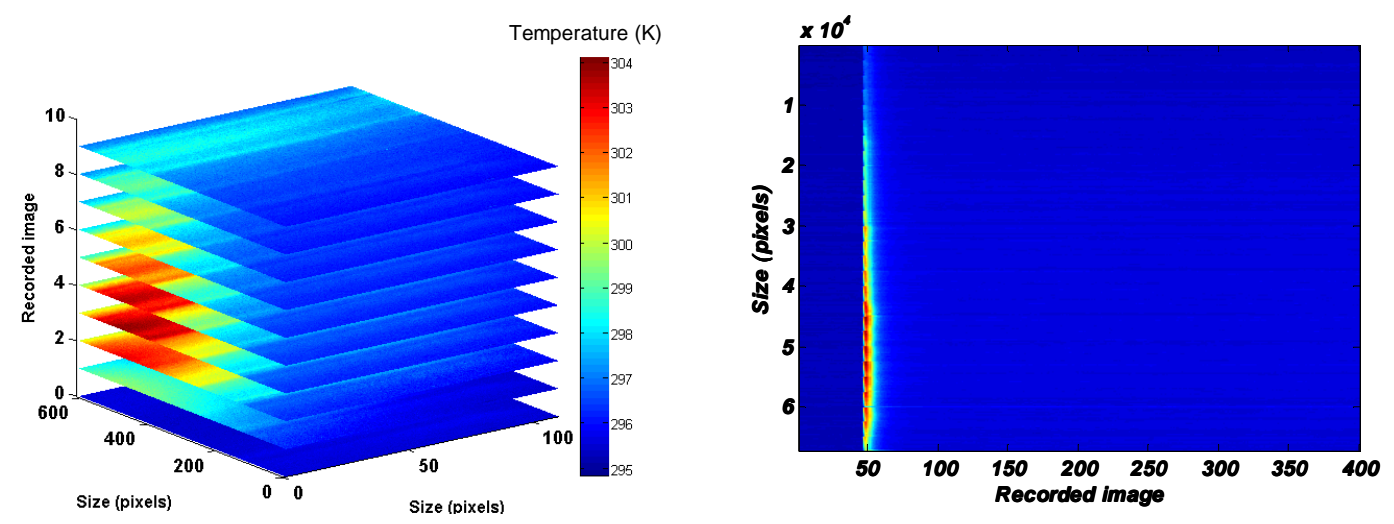

Fig. 2. (a) Example of thermal data recorded at nine different instants by the FLIR® camera for a sample surface excited by all the flashes (b) 2D raster-like matrix of thermal data. The color bar scale spans from 295 to $304 \mathrm{~K}$.

\subsection{Data processing: a Partial Least Squares Regression approach (PLSR)}

Partial Least Squares (also known as Projection to Latent Structure) is a popular method for modeling industrial applications. It was developed by Wold et al. [14]. PLS has been used as a popular tool for regression in many fields such as medicine [15], social science [16] but especially in chemistry [17].

For our application, the PLS regression (PLSR) looks for modeling a linear relationship between the predictor $X$ (the 2D thermal response matrix) and a predicted $Y$ (a column-vector composed of a time series). The goal of this technique is to create orthogonal scores vectors (also called latent vectors or component) while maximizes the covariance between $X$ and $Y$ [18]. In this study, the application of PLSR to the pulsed thermography data arises from the necessity to decompose the 


\subsection{1/qirt.2016.088}

thermal data obtained during the inspection into a new sequence of images less contaminated by noise and excitation inhomogeneity while maintaining physical coherency [10]. This advanced signal processing tool, based on a regression method, is used to decompose the thermal data (represented by the matrix $X$ ) at each instant into a set of latent variables in order to identify and select the effective component corresponding to the coating thickness heterogeneity. In this study, the partial least square regression is iterated to generate a set of scores, loadings and errors matrices as following (Eq. (1) and (2)):

$$
\begin{aligned}
& X=T P^{T}+E \\
& Y=T Q^{T}+F
\end{aligned}
$$

where $T$ are the score matrix, $P$ and $Q$ are matrices of coefficients (called also loading matrices), $E$ and $F$ are the error matrices, and $T$ denote the matrix transpose. To calculate the loading and scores, the latent variables are computed by iterative applications of the singular value decomposition (SVD) which states that a matrix $M$ can be written as the product of three matrices: $U, S$ and $V$ (Eq. (3)):

$$
M=U S V^{T}
$$

In this study, we are interested only in latent variables or loading, which describe the observations and explain the largest portion of the covariance between matrix $X$ and univariate $Y$-block [19].

The PLS-SVD algorithm, detailed by Abdi [20], carries out the simultaneous decomposition of matrix $X^{T} Y$ through the computation of scores, weights, loadings and inner-coefficients. The general step of this algorithm are:

The inputs $X$ and $Y$ are first mean-centered and variance scaled.

Step1: find the eigenvectors $U$ and $V$ by calculating the SVD decomposition of $X^{T} Y$

Step 2: compute the normalized vector $t=\frac{X^{T} U}{\left\|X^{T} U\right\|}$ and the following elements:

Step 3: $u=Y V$

Step 4: $p=X^{T} t$

Step 5: $b=u^{T} t$

Storing these vectors in the column of correspondent matrix

After calculating the score and the loading of the first latent variable, $X$ and $Y$ residuals are calculated:

Step 6: $X=X-t p^{T}$

Step 7: $Y=Y-t b c^{T}$

Step8: Repeat steps 1 to 7 replacing $X$ and $Y$ with their residuals for each desired factor.

It assumed that the matrices $U U^{T}$ and $V^{T} V$ are an identity matrix.

This show that the first weight vector $U$ is the first right singular vector of the matrix $X^{T} Y$. Similarly, the first weight vector $V$ is the left singular vector of $X^{T} Y$. The same argument shows that the first vectors $t$ and $u$ are the first eigenvectors of $X X^{T} Y Y^{T}$ and $Y Y^{T} X X^{T}[21]$.

\section{Results and discussions}

The proposed approach aims to find component from $X$ that are relevant for $Y$. These components incorporate information on both $X$ and $Y$ in the definition of the scores and loadings. In fact, the score and loading are chosen in such a way to explain as much as possible of the covariance between $X$ and $Y$ [22]. Figure 2 shows the plot of ten loading with respect to its variance explained in $X$. It can be noted that the second loading represent a higher variance explained in $X$ and a significant signal scores. This loading corresponds to the mean thermal response without noise. 


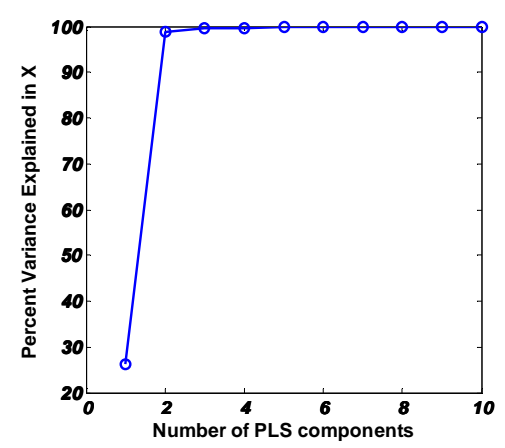

(a)

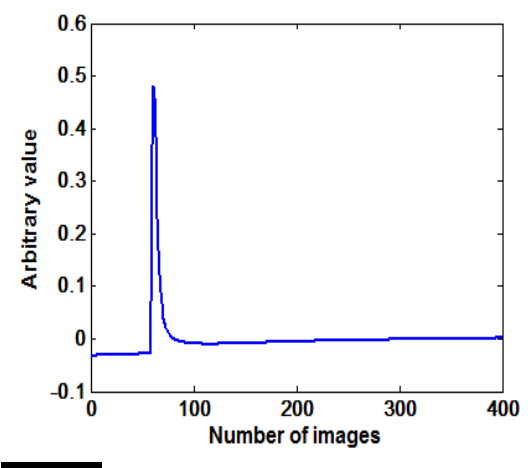

(b)

Fig. 2. (a) Validation of the PLSR model: percent variance explained in Y. (b) the score of loading 2 which represent the most significant latent variable. It represents the thermal response without noise.

As shown in Figure 3, the thermal images extracted from the raw data at the instant time for which the excitation is maximal highlight clearly the three bands of the coating. However, variations in the raw thermal responses when changing excitation conditions are also observed. Moreover, the thermal responses are not uniform inside each band of the coating. These variations are due to the non-uniform excitation of flash sources. Figure 3 shows also the results obtained by the application of the proposed PLSR technique. This advanced signal processing method permits to remove the variability between the thermal images of the different considered experimental configurations of flash sources.

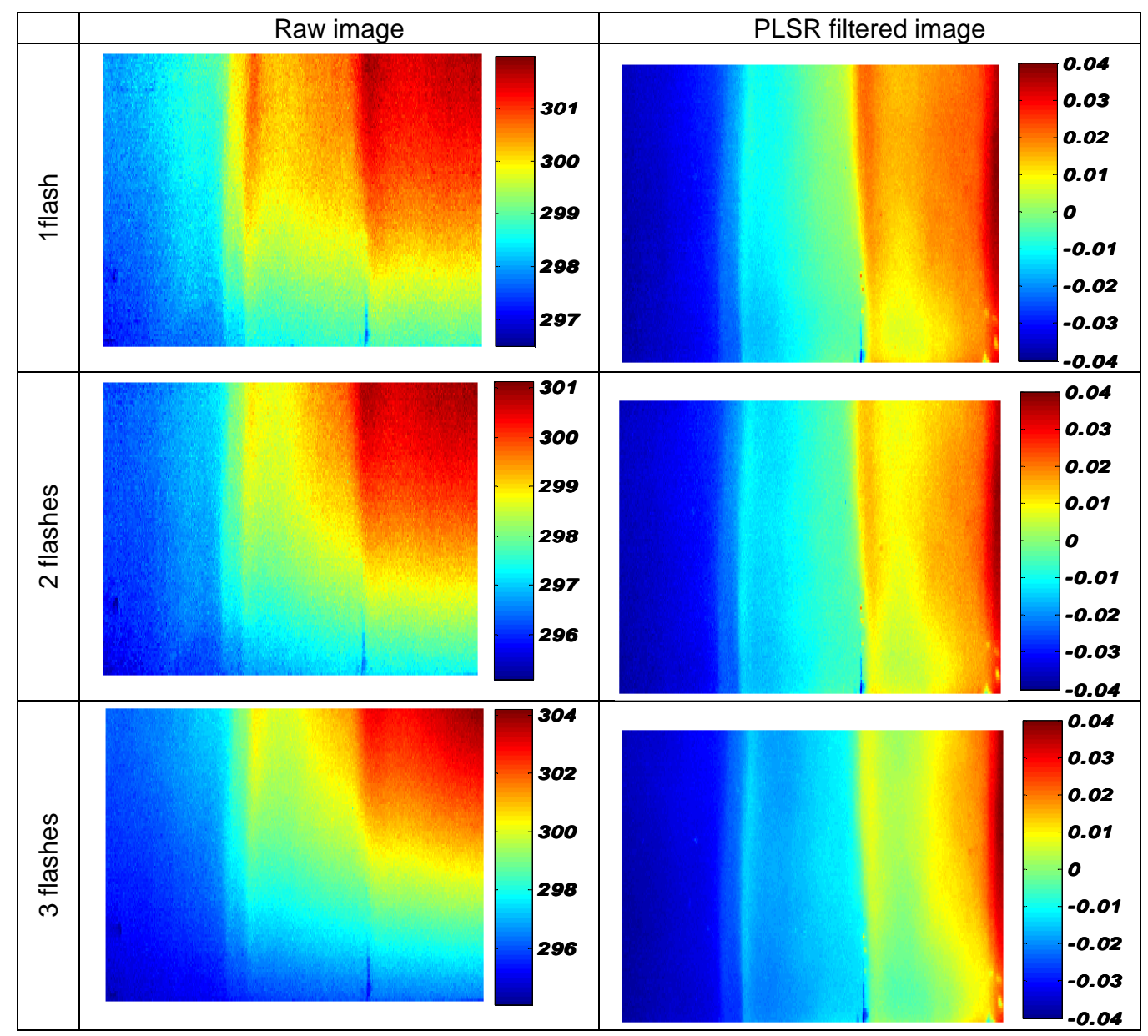




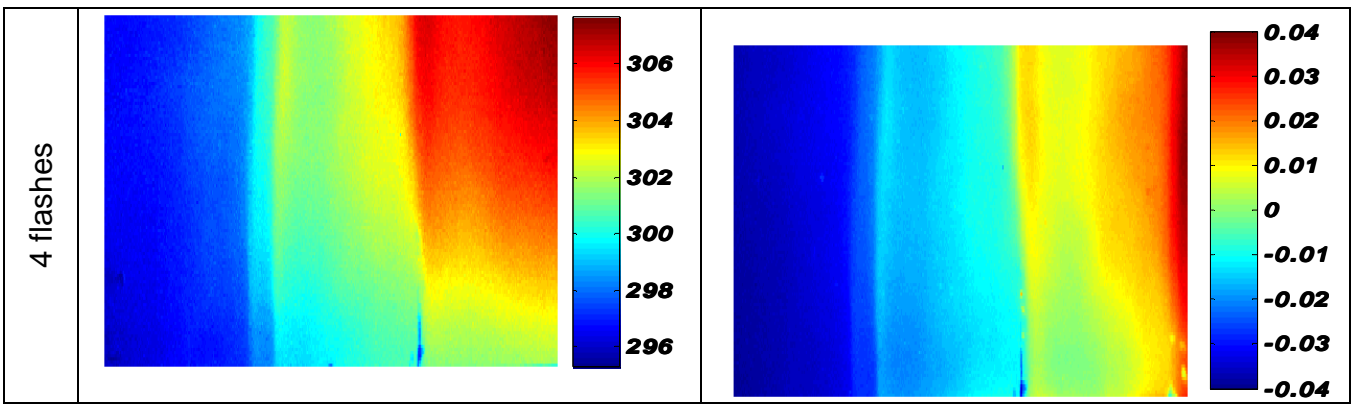

Fig. 3. Thermal images at maximum heating for different experimental configurations of excitation sources obtained before (a) and after (b) the PLSR processing.

For a better comparison, figure 4 shows the thermal profiles, i.e. thermal variations for a row of the thermal image, before (raw) and after the PLSR processing. Unlike the raw profiles, the treated ones are insensitive to experimental conditions.
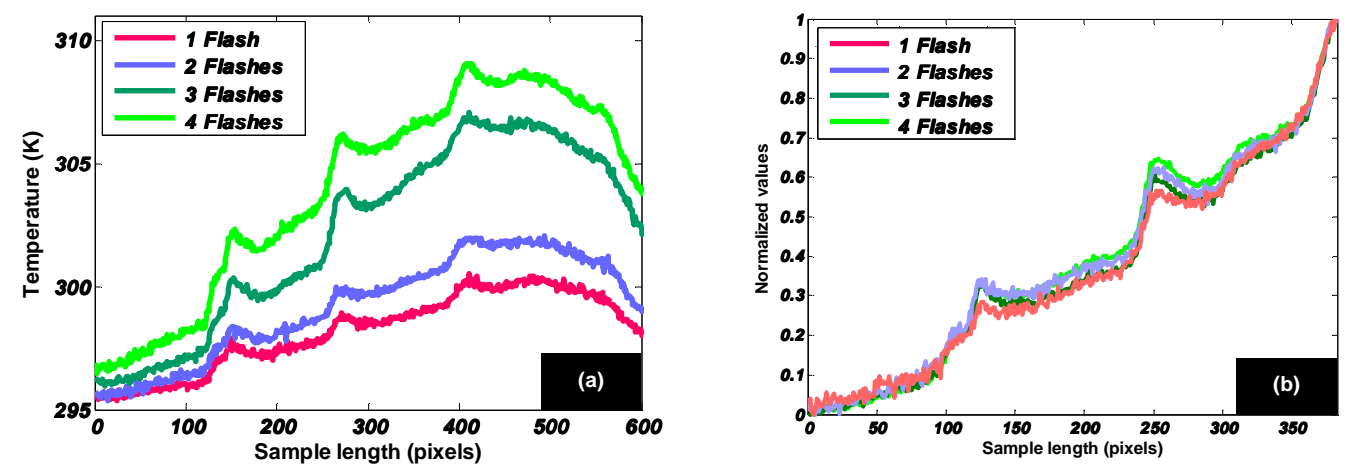

Fig. 4. Thermal responses for different experimental configurations of excitation sources obtained before (a) and after (b) the PLSR processing.

For industrial application, it is useful to determine the relationship between the temperature recorded by the infrared camera and the measured thicknesses. The paint thickness variations of our samples were evaluated with the Eddy current method in the same position of the extracted thermal profile. A linear regression model between the Eddy current measurements and normalized temperatures obtained after PLSR filtering is then established, which gives the following relation:

$$
\mathrm{T}=42,581 \mathrm{~N}+54,791 \quad \text { with correlation coefficient } \mathrm{r}^{2}=0.93
$$

where $\mathrm{N}$ is the normalized thermal response after PLSR filtering and T is the paint coating thickness in $\mu \mathrm{m}$ estimated By the Eddy current method. This linear relation demonstrates clearly that the processed active infrared thermography technique is as sensitive as Eddy current method. However, the active infrared thermography is faster, more spatially resolved, and more efficient to detect the heterogeneity of coating thickness by providing quickly a 2D measure of a large area. Furthermore, by applying the proposed PLSR filtering technique, this relationship is independent of experimental configurations, especially positions and powers of source excitations. Finally, we must note that this calibration was validated only for coating thickness ranging in $[50 \mu \mathrm{m}, 80 \mu \mathrm{m}]$ and in the case of a bi-layered sample (epoxy /stainless steel). Further works will be focused to extend this relationship to a larger coating thickness domain and to validate the proposed technique to others coating and substrate materials.

\section{Conclusion}

The proposed approach based on pulsed thermography system and a partial least squares regression approach preceded by a preprocessing of thermal data presents an effective valuable solution as a NDT technique for enhancing the evaluation of coating thickness heterogeneity on metal plates. Future works will focus on the validation of the developed approach for 


\subsection{1/qirt.2016.088}

non-conductive substrates materials as well (PVC, polypropylene...) and to extend this study to a larger coating thickness range.

\section{Acknowledgements}

The research for this paper was financially supported by the Communauté d'Agglomération de Châlons-en-Champagne, France.

\section{Bibliography}

[1] Meriaudeau, F., "Real time multispectral high temperature measurement: Application to control in the industry" Image and Vision Computing, Vol. 25, Issue. 7, pp. 1124-1133, 2007.

[2] Luong M.P., "Fatigue limit evaluation of metals using an infrared thermographic technique", Mechanics of Materials, Vol. 28, Issue. 1-4, pp. 155-163, 1998.

[3] Aoyagi M., Hiraguri T., Ueno T., "Nondestructive analysis of uneven paint coatings by dynamic heat conduction following flash heating", J. Coat. Technol. Res., Vol.11, No. 3, pp. 311-318, 2014. http://dx.doi.org/10.1007/s11998-0139537-8.

[4] Chatterjee K., Tuli S., "Image Enhancement in Transient Lock-In Thermography Through Time Series Reconstruction and Spatial Slope Correction”, IEEE Trans. Instrum. Meas. Vol. 61, No. 4, pp. 1079-1089, 2012.

[5] Ibarra-Castanedo C., Gonzàlez D., Klein M., Pilla M., Vallerand S., Maldague X., "Infrared Image Processing and Data Analysis", Infrared Physics and Technology, Vol. 46, Issue. 1-2, pp. 75-83, 2004.

[6] Benítez H., Ibarra-Castanedo C., Bendada A., Maldague X., Loaiza H., Caicedo E., "Defect Characterization in Infrared Nondestructive Testing with Learning Machines", NDT\&E international, Vol. 42, Issue. 7, pp. 630-643, 2009.

[7] Benitez H., Maldague X., Ibarra-Castanedo C., Loaiza H., Bendada A., Caicedo E., "Modified Differential Absolute Contrast using Thermal Quadrupoles for the Nondestructive Testing of Finite Thickness Specimens by Infrared Thermography", 2006 Canadian Conference on Electrical and Computer Engineering, Ottawa, Ont., pp. 1039-1042, 2006. doi: 10.1109/CCECE.2006.277741.

[8] Grinzato E., Bison P., Marinettti S., Vavilov V., "Thermal NDE enhanced by 3D Numerical Modeling applied to Works of Art", Insight: Non-Destructive Testing and Condition Monitoring, Vol. 43, no. 4, pp. 254-259.

[9] Restrepo A., Loaiza $H$. "Non-uniform heating compensation for sequences of thermal images using median filtering". DYNA journal, Vol. 80, no. 182, pp. 74-82, 2013.

[10] López F., Nicolau V., Maldague X, Ibarra-castanedo C., "Multivariate Infrared Signal Processing by Partial Least Squares Thermography", Proceedings of the VIlth International Workshop p Advances in Signal Processing for NDT, Non Destruction Evaluation of Materials, (Canada) Quebec, 2013.

[11] Vrabie V., Perrin E., Bodnar J-L., Mouhoubi K., Detalle V., "Active IR thermography processing based on higher order statistics for non-destructive evaluation", 20th European Signal Processing Conference Bucharest, Romania, pp. 2731, 2012.

[12] Ibarra-Castanedo C., Genest M., Piau J-M., Guibert S., Bendada A., Maldague X.P.V., "Active infrared thermography techniques for the non-destructive testing of materials", Ultrasonic and Advanced Methods for Nondestructive Testing and Material Characterization, pp. 325-348, 2007. doi: 10.1142/9789812770943_0014.

[13] Mezghani S., Perrin E., Bodnar J.L, Cauwe B., Vrabie V., "Multiscale analysis of thermography imaging dynamic for sol-gel coating discrimination", Proceedings of 12th Quantitative InfraRed Thermography conference, paper QIRT2014199, Bordeaux (France), 2014.

[14] Wold H., "Estimation of principal component and related models by iterative least squares". In: P. R. Krishnaiah (Ed.), Multivariate Analysis, Academic Press, New York, pp. 391-420, 1966.

[15] Worsley K.J., "An overview and some new developments in the statistical analysis of PET and fMRI data", Human Brain Mapping, Vol. 5, no.4, pp. 254-258, 1997.

[16] Hulland J.S., "Use of partial least squares (PLS) in strategic management research:A review of four recent studies", Strategic Management Journal, Vol. 20, no. 2, pp.195-204, 1999.

[17] Wold S., Sjöström M., Eriksson L., "PLS-regression: a basic tool of chemometrics", Chemometrics and Intelligent Laboratory Systems", Vol. 58, no. 2, pp. 109-130, 2001. http://dx.doi.org/10.1016/S0169-7439(01)00155-1.

[18] Russolillo G., "Non-Metric Partial Least Squares”, Electronic Journal of Statistics, Vol. 6, pp. 1641-1669, 2012. ISSN: 1935-7524, doi: 10.1214/12-EJS724.

[19] Abdi H., Williams L.J., "Chapter 23 Partial Least Squares Methods: Partial Least Squares Correlation and Partial Least Square Regression”, Methods Mol. Biol., Vol. 930, pp. 549-579, 2012.

[20] Abdi H., "Partial least squares regression and projection on latent structure regression (PLS Regression)", Wiley Interdisciplinary Reviews: Computational Statistics, Vol.2, Issue. 1, pp. 97-106, 2010.

[21] Abdi H., "Partial least square regression (PLS regression)", In: Lewis-Beck M., Bryman A., and Futing. T et al. (Eds), Encyclopedia of social sciences research methods, Sage, Thousand oaks, CA, pp. 792-795, 2003.

Mevik B. H., Wehrens R., "The PLS package: principal component and partial least squares regression in R", J. Stat. Softw., Vol. 18, Issue.2, pp. 1-24", 2007. 